Bull. Mater. Sci., Vol. 21, No. 4, August 1998, pp. 283-286. (C) Indian Academy of Sciences.

\title{
Electron cyclotron resonance (ECR) plasma-enhanced chemical vapour deposition of silicon dioxide on strained-SiGe films using tetraethylorthosilicate
}

\author{
L K BERA, S K RAY ${ }^{\dagger}$ H D BANERJEE* and C K MAITI \\ Department of Electronics and ECE, 'Department of Physics, *Materials Science Centre, Indian Institute of \\ Technology, Kharagpur 721302 , India \\ MS received 13 February 1998
}

\begin{abstract}
Silicon dioxide films on strained $\mathrm{Si}_{1-x} \mathrm{Ge}_{x}$ have been deposited by electron cyclotron resonance (ECR) plasma-enhanced chemical vapour deposition technique using tetraethylorthosilicate (TEOS) at room temperature. The deposition rate as a function of time and substrate temperature has been studied. MOS capacitors fabricated using deposited oxides have been used to characterize the electrical properties of silicon dioxide films. Deposited oxide film shows its suitability for microelectronic applications.
\end{abstract}

Keywords. Silicon-germanium; silicon dioxide; ECR plasma deposition; TEOS.

\section{Introduction}

Strained-SiGe/Si alloy has attracted much attention from the point of view of novel bandgap engineered devices as well as for its attractive optical and electronic properties (Jain and Hayes 1991). A high quality dielectric layer formation is an integral part of the MOSFET fabrication. Conventional thermal oxidation and rapid thermal oxidation revealed selective oxidation of $\mathrm{Si}$ and pile-up of $\mathrm{Ge}$ atoms at the oxide/SiGe interface resulting in an unacceptably high density of interface states (LeGoues et al 1989; Nayak et al 1990). To get rid of this problem, silicon cap layers are commonly used. However, high temperature $\left(>700^{\circ} \mathrm{C}\right)$ processing is not possible for strained SiGe layers as it causes strain relaxation. It also introduces additional dislocations and stacking faults at the interface. Therefore, the formation of dielectric films on strained-SiGe layers at low temperature is required to achieve high quality interfaces and to prevent strain relaxation in pseudomorphic alloys. We have reported low-temperature $\left(<200^{\circ} \mathrm{C}\right)$ oxidation of strainedSiGe films by non-ECR microwave plasma method (Mukhopadhyay et al 1994, 1996), and the results of our studies on the electrical properties of thin $(\approx 120 \AA)$ $\mathrm{SiO}_{2}$ films deposited on strained-SiGe layers using TEOS at room temperature by electron cyclotron resonance (ECR) microwave plasma. The electrical properties of the oxide films have been characterized using a MOS structure.

*Author for correspondence

\section{Experimental}

A schematic view of the microwave-ECR plasma system used in our experiments is shown in figure 1 . The variable microwave power $(50-1000 \mathrm{~W}, 2.45 \mathrm{GHz})$ generated by a magnetron was guided through a waveguide to the ECR cavity. The waveguide was separated from the ECR cavity by a quartz bell jar. The ECR condition was adjusted by a three-stub tuner and accordingly the electromagnetic mode could be changed. In order to reproduce the process, it is important to choose a distinct electromagnetic mode that could provide a stable plasma. The ECR source was surrounded by magnetic solenoids to get the resonance condition. Sufficient electron kinetic energy could be obtained by introducing a steady magnetic field $B=875 \mathrm{G}$ for the electron cyclotron frequency $\omega_{\mathrm{c}}=e B / m c$ in resonance chamber. The stainless steel sample holder was fitted with an electrical heater (up to $600^{\circ} \mathrm{C}$ ) to raise the substrate temperature. The reflected power was kept below $10 \%$ of the forward microwave power to avoid damage to the magnetron.

Nominally undoped strained $\mathrm{Si}_{1-x} \mathrm{Ge} / \mathrm{Si}$ layers were grown on $p$-Si $\langle 001\rangle(5-10 \Omega-\mathrm{cm})$ substrates using a gas source molecular beam epitaxy (GSMBE) at $700^{\circ} \mathrm{C}$. An undoped $\mathrm{Si}$ buffer layer, $500 \AA$, was used to provide a good quality surface for subsequent growth of the $\mathrm{SiGe}$ epilayer. The SiGe epilayer grown had a Ge concentration of $26 \%$ (thickness $300 \AA$ ). Though the purity of disilane was more than $99.99 \%$, it showed the following impurity levels: silane $<20 \mathrm{ppm}, \mathrm{H}_{2}<10 \mathrm{ppm}, \mathrm{H}_{2} \mathrm{O}<0.1 \mathrm{ppm}$, hydrocarbons $<1.0 \mathrm{ppm} . \mathrm{N}_{2}, \mathrm{O}_{2}, \mathrm{CO}_{2}$ and trisilane were 
nondetectable. Likewise, though the purity of germane was more than $99.99 \%$, it showed the following impurity levels: $\mathrm{O}_{2}<20 \mathrm{ppm}, \mathrm{N}_{2}<40 \mathrm{ppm}, \mathrm{H}_{2}<30 \mathrm{ppm}$, $\mathrm{CO}_{2}<10 \mathrm{ppm}$ and $\mathrm{H}_{2} \mathrm{O}<10 \mathrm{ppm}$.

The oxide films were deposited in the temperature range of $30-400^{\circ} \mathrm{C}$. All the samples were subjected to a standard cleaning schedule followed by dipping in dilute HF just before loading inside the chamber. In a typical deposition process, the system was first evacuated to $10^{-5}$ Torr and then TEOS was introduced into the system independently from a bubbler kept in a constant temperature $\left(45^{\circ} \mathrm{C}\right)$ bath with nitrogen as the carrier gas. For the prevention of condensation of TEOS in the line, a heated delivery system was employed.

Oxide thicknesses were both measured by a single wavelength (6328 $\AA$ ) ellipsometer (Gaertner L-117) as well as calculated from the oxide capacitances in accumulation. The breakdown field and interface charge trap density were obtained from $J-E$ and $C-V$ measurements on MOS capacitors. For the electrical measurements of MOS structures, an HP4061A computerized semiconductor test system was used comprising a multifrequency LCR meter (HP4275A), a picoammeter/dc voltage source (HP4140B) and a switching subsystem.

\section{Results and discussion}

The plot of deposited film thickness vs deposition time is shown in figure 2. A linear relationship was observed over the time range investigated. Similar characteristics

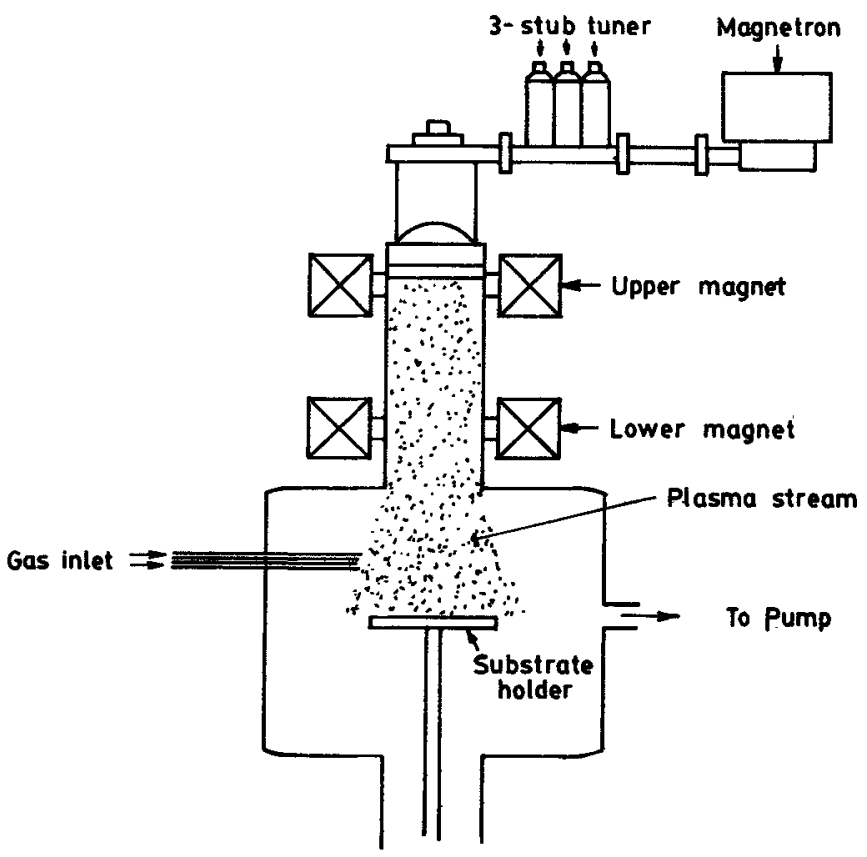

Figure 1. Schematic view of the microwave-ECR plasma system. for bulk-Si have been reported by Secrist and Mackenzie (1966). Connell and Gregor (1965) showed that in case of bulk-Si, the deposition rate decreased when the deposition time was varied from $40 \mathrm{sec}$ to $4 \mathrm{~min}$ and finally it became constant at the end of this period. The existence of such an initial period of decrease in the deposition rate in all glow discharge reactions is expected, since initially the plasma density changes due to the interaction with the reacting species present in the chamber, and

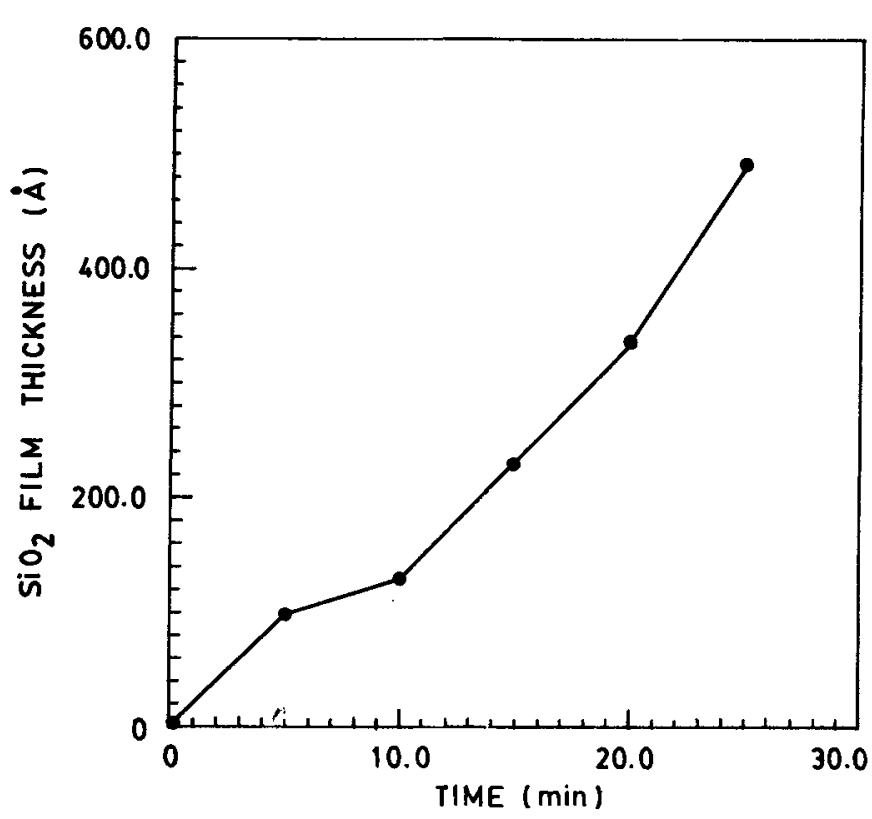

Figure 2. The plot of deposited film thickness vs time.

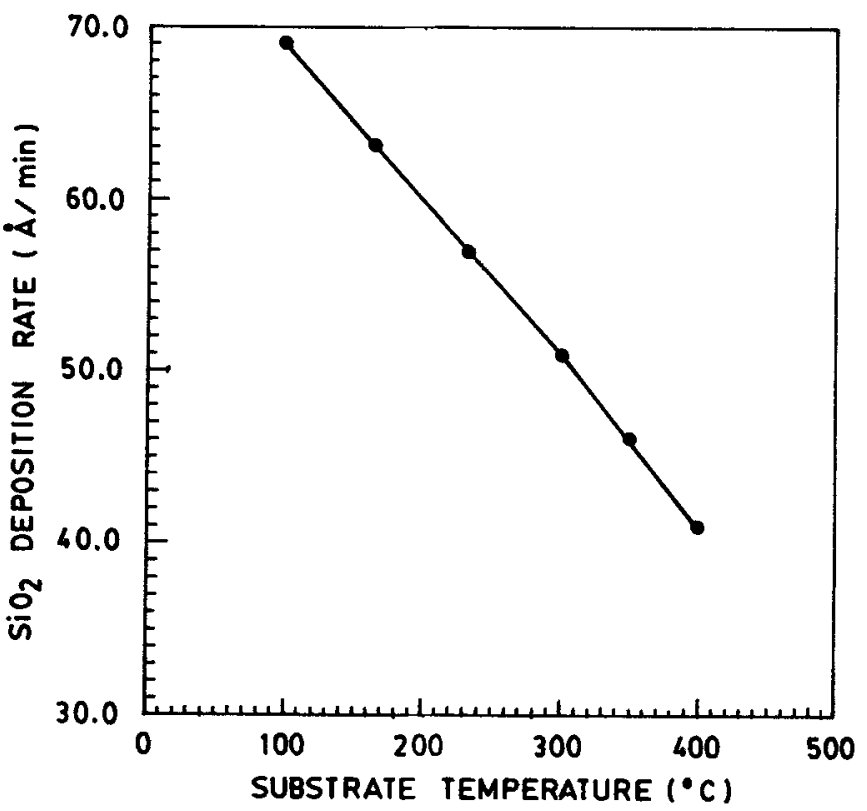

Figure 3. The plot of deposition rate vs substrate temperature. 
probably this initial period represents the time required to attain equilibrium in the plasma.

The film thickness as a function of substrate temperature shows that the deposition rate decreases with increase in substrate temperature (figure 3). This decrease in the deposition rate is characteristic of an adsorption-controlled process, whereby for the free energy of the system to decrease, heat must be evolved, and the rate of such an exothermic process generally decreases with increasing temperature. From an atomistic viewpoint, in this study, the 'silicon-oxygen species' could be imagined to possess a mean lifetime in which to select a site on the substrate surface. Therefore the temperature is raised, more kinetic energy would be imparted to the species, and hence the mean life time for adsorption decreases (Secrist and Mackenzie 1966).

Figure 4 shows typical $1 \mathrm{MHz}$ capacitance-voltage $(C-V)$ characteristics obtained for an as deposited sample. The curve exhibits a negative flatband voltage, indicating the existence of positive charges in the films. Fixed oxide charge density $\left(Q_{\mathrm{r}} / q\right)$ for the sample is $2.9 \times 10^{11} \mathrm{~cm}^{-2}$. The $C-V$ and conductance-voltage $(G-V)$ characteristics of a MOS capacitor at different frequencies are shown in figure 5 . In the accumulation region, the $C-V$ curve shows a little dispersion with a higher capacitance value at $100 \mathrm{kHz}$. The $G-V$ characteristics, on the other hand, show a frequency dispersion in the accumulation region where a higher conductance value was obtained for $1 \mathrm{MHz}$. The interface state density, $D_{i t}$ was calculated from the high frequency $C-V$ and $G-V$ characteristics using Hill's method (Hill and Coleman 1980). At the midgap, the interface state density value obtained is $5.8 \times 10^{11} \mathrm{~cm}^{-2} \cdot \mathrm{eV}^{-1}$.

The $I-V$ curves were measured in the accumulation

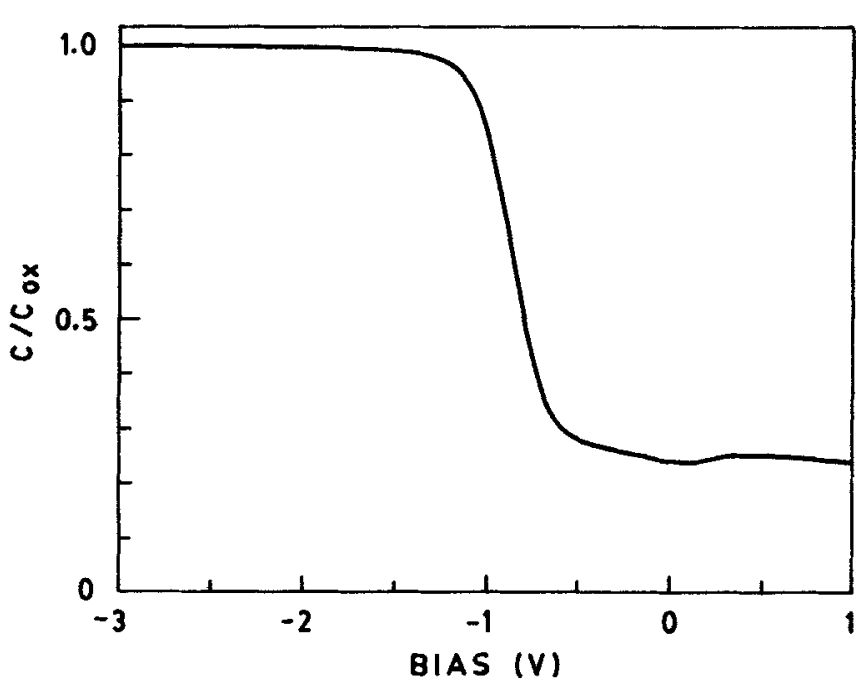

Figure 4. High frequency $C-V$ characteristics of microwave ECR (TEOS) plasma deposited oxides on strained $S i_{(1.74} \mathrm{Ge}_{026}$ samples. regime for the as deposited MOS capacitor. Figure 6 shows typical $J-E$ characteristics of the MOS capacitor. The resistivity value of the oxide film is about $3.1 \times 10^{16} \Omega$-cm. The critical field $\left(E_{c}\right)$, defined as the field required to reach an injection current density of $1 \mathrm{nA} \mathrm{cm}{ }^{-2}$ (DiMaria et al 1980), can be deduced from the $J-E$ characteristic (see figure 6). In the high electric field region, a linear relation was obtained between $\ln J / E$ and $\sqrt{E}$. Assuming a Pool-Frenkel mechanism, the characteristic $J-E$ is described by the following expression:

$$
J=a E \exp \left[\frac{1}{r k T} \sqrt{\frac{q^{3} E}{\pi \varepsilon_{0} \varepsilon_{\mathrm{d}}}}\right],
$$

where $a$ is a constant, $k$ the Boltzmann constant, $T$ the temperature and $r$ is a constant having a value between 1 and 2 depending on the traps in the insulator. For our samples, $r=1.6$ and a value $\varepsilon_{\mathrm{d}} \approx 4.04$ was deduced for the high-frequency dielectric constant. The breakdown field strength with a current criteria of $1 \mu \mathrm{A} \mathrm{cm}^{-2}$ is calculated to be $7.0 \mathrm{MV} / \mathrm{cm}$.

\section{Conclusion}

The study has demonstrated the low-temperature deposition of thin oxides $(\approx 120 \AA)$ on strained- $\mathrm{Si}_{1-x} \mathrm{Ge}_{x}$ films,

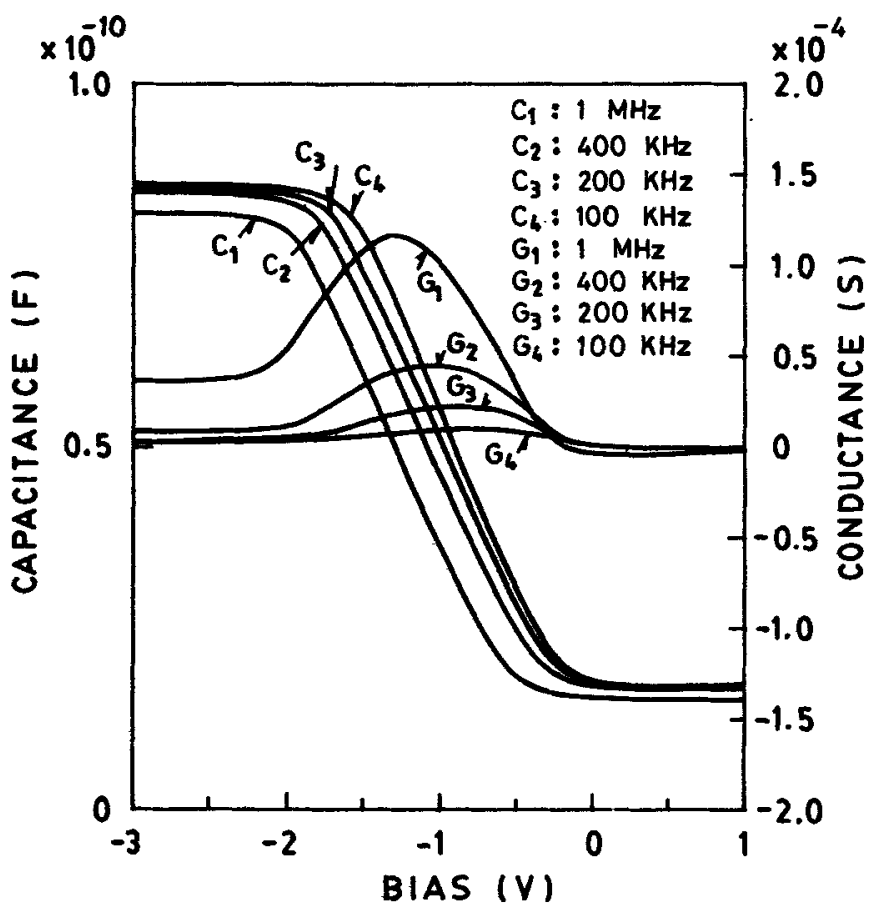

Figure 5. Measured conductance, $G$ and capacitance, $C$ vs applied voltage characteristics at different frequencies for microwave-ECR (TEOS) plasma deposited oxides on strained $\mathrm{Si}_{0.74} \mathrm{Ge}_{0.26}$ samples. 


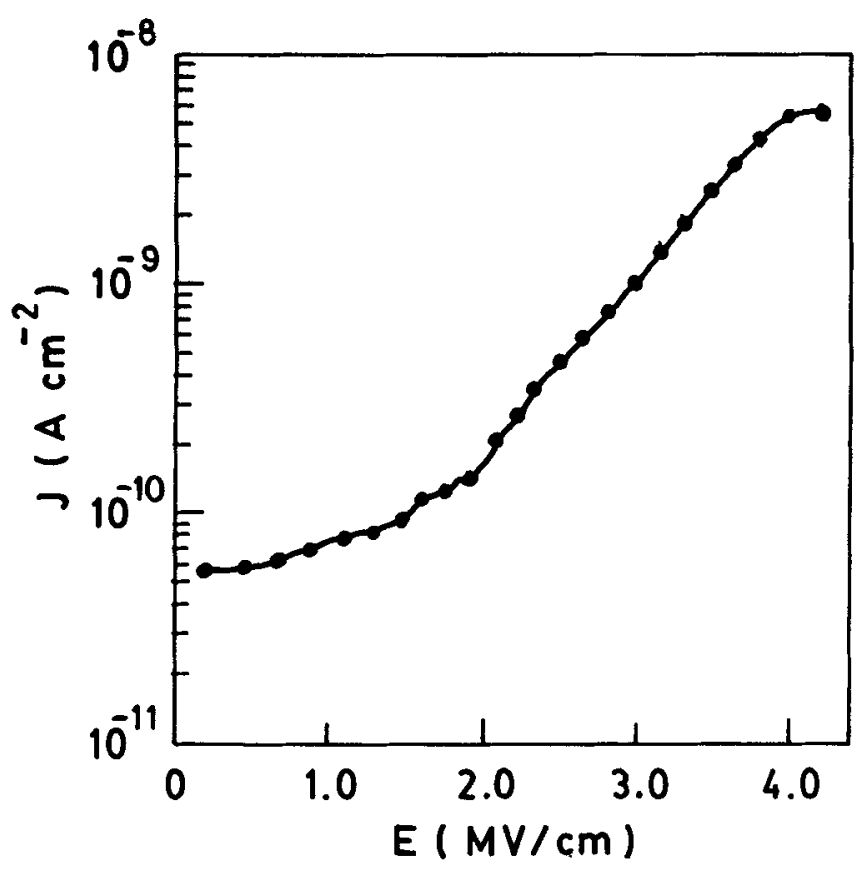

Figure 6. Leakage current density $(J)$ as a function of the electric field $(E)$ for microwave ECR (TEOS) plasma deposited oxides on strained $\mathrm{Si}_{0.74} \mathrm{Ge}_{026}$ samples.

using TEOS by microwave ECR plasma. The electrical properties of the deposited oxides are reported. The oxide films on strained SiGe samples exhibited a high resistivity $\left(3.1 \times 10^{16} \Omega-\mathrm{cm}\right)$ and a critical field of about $2 \mathrm{MV} \mathrm{cm}^{-1}$.
Fixed oxide charge density $\left(Q_{\mathrm{r}} / q\right)$ and interface trap density $\left(D_{\mathrm{i} i}\right)$ for the oxides deposited on strained-Si $\mathrm{S}_{1-x} \mathrm{Ge}_{x}$ samples in microwave ECR plasma are $2.9 \times 10^{11} \mathrm{~cm}^{-2}$ and $5.8 \times 10^{11} \mathrm{~cm}^{-2} \cdot \mathrm{eV}^{-1}$, respectively.

\section{Acknowledgements}

We thank Dr D K Nayak and Prof. Y Shiraki, University of Tokyo, Japan, for preparing the strained-SiGe films used in this study.

\section{References}

Connell R A and Gregor L V 1965 J. Electrochem. Soc. 112 1199

DiMaria D J, Ghez R and Wong D W 1980 J. Appl. Phys. 51 4830

Hill W A and Coleman G C 1980 Solid State Electron. 23987

Jain S C and Hayes W 1991 Semicond. Sci. Technol. 6547

LeGoues F K, Rosenberg R and Meyerson B S 1989 Appl. Phys. Lett. 54644

Mukhopadhyay M. Ray S K, Maiti C K, Nayak D K and Shiraki Y 1994 Appl. Phys. Lett. 65895

Mukhopadhyay M, Ray S K, Nayak D K and Maiti C K 1996 Appl. Phys. Lett. 681262

Nayak D K, Kamjoo K, Park J S, Woo J C S and Wang K L 1990 Appl. Phys. Lett. 57369

Secrist D R and Mackenzie J D 1966 J. Electrochem. Soc.: Solid-State Sc. \& Tech. 113914 